ORIGINAL ARTICLE

\title{
Blood transfusion in patients treated with surgery for necrotizing enterocolitis
}

\author{
Zulfiqar Ahmed ${ }^{1}$, Logan Danielson ${ }^{2}$, Rashad Albeiruti ${ }^{3}$, Ronald Thomas ${ }^{4}$ \& Michael Klein ${ }^{2}$ \\ 1 Anesthesia Associates of Ann Arbor, Oakwood Hospital and Medical Center, Dearborn, MI, USA \\ 2 Department of Surgery, Children's Hospital of Michigan, Detroit Medical Center, Wayne State University, Detroit, MI, USA \\ 3 Department of Anesthesiology, University of Michigan, Ann Arbor, MI, USA \\ 4 Children's Research Medical Center, Children's Hospital of Michigan, Detroit Medical Center, Detroit, MI, USA
}

\begin{abstract}
Keywords
neonatology; anesthesiology; hemodynamics; preterm infants; emergencies; critical care

\section{Correspondence \\ Zulfiqar Ahmed, Anesthesia Associates of Ann Arbor, Oakwood Hospital and Medical Center, 10801 Oakwood Blvd Dearborn, MI 48124, USA \\ Email: zahmedz@yahoo.com}

Section Editor: Neil Morton

Accepted 16 June 2014

doi:10.1111/pan.12485

\begin{abstract}
Summary
Background: Necrotizing enterocolitis (NEC) is a common surgical emergency in premature infants and has high morbidity and mortality. Intraoperative treatment with fluid and transfusions may be difficult.

Objectives: We evaluated risk factors for patients who needed transfusion with packed red blood cells during surgery for necrotizing enterocolitis with bowel resection.

Methods: Retrospective medical and anesthetic record review of 206 patients who had necrotizing enterocolitis during 10 years at a pediatric referral center.

Results: In the 206 patients who had necrotizing enterocolitis, 88 patients $(43 \%)$ had exploratory laparotomy, 67 with bowel resection. Of these, 50 needed transfusion of packed red blood cells in comparison with 17 who did not. Upon univariate analyses, patients who received packed red blood cells during surgery had significantly longer mean duration of surgery, lower preoperative hemoglobin and hematocrit, higher postoperative hemoglobin and hematocrit, more platelet transfusions, and greater estimated blood loss than patients who did not receive packed red blood cells during surgery. A binary logistic regression procedure revealed that the need for preoperative hemoglobin was found to be a significant risk factor for transfusion of packed red blood cells (odds ratio $=0.55 ; 95 \% \mathrm{CI}=0.36-0.84 ; P=0.001$ ).

Conclusion: Patients having necrotizing enterocolitis with bowel resection are more likely to need preoperative transfusion with packed red blood cells, which may be more necessary when surgery is longer, preoperative hematocrit is lower, and estimated blood loss is higher.
\end{abstract}

\section{Introduction}

Necrotizing enterocolitis is a common surgical emergency in neonates (1). This condition causes major morbidity and mortality in neonates, especially premature neonates. NEC is characterized by partial or full thickness intestinal ischemia usually in the terminal ileum but may involve the entire bowel (Necrotozing Entercolitis Totalis). Known risk factors include prematurity, neonatal stress, formula feeding, and surgery in newborn period. (i) Clinical diagnosis is made with signs and symptoms combined with radiologic findings as discussed in Bell classification. According to the published reports, the incidence of NEC in newborn infants is between $4 \%$ and $7 \%$. The initial treatment of NEC is nonsurgical with fluids, ventilatory support, ionotropes, and antibiotics among other measures. A subset of these patients undergoes surgical exploration of the abdomen $(2,3)$. Surgical treatment options include primary peritoneal drains or laparotomy with bowel resection and creation of an ileostomy $(4,5)$. The frequency of mortality resulting from necrotizing enterocolitis is from $20 \%$ to $30 \%$, highest in the smallest infants and those requiring surgery $(1,6,7)$. 
Anesthetic considerations in the operative treatment of necrotizing enterocolitis include fluid treatment and blood product transfusion. This treatment has been based on general principles of the treatment of newborn and premature infants. We hypothesized that bowel resection may increase the risk of needing intraoperative transfusion of packed red blood cells in patients having exploratory laparotomy for necrotizing enterocolitis. The purpose of this study was to evaluate risk factors for needing transfusion of packed red blood cells in patients having surgery for necrotizing enterocolitis.

\section{Methods}

\section{Patients}

The Neobase registry at the Children's Hospital of Michigan collected data prospectively about every patient who was admitted to the neonatal intensive care unit. From this registry, we identified 206 patients who had necrotizing enterocolitis during 10 years (October 2001 to June 2010). The registry data, medical records, and anesthesia records of these 206 patients were reviewed retrospectively. All patients who had exploratory laparotomy for necrotizing enterocolitis were included. The Wayne State University Institutional Review Board approved this study.

\section{Evaluation}

Patients were divided into two groups: patients who received or did not receive intraoperative transfusion of packed red blood cells. We extracted the following information: gestational age and body weight at birth; age and body weight at surgery; preoperative and postoperative hematologic and chemistry data; type of surgical procedure (exploration alone or bowel resection); and total amount of any blood products transfused. The primary outcome was whether or not a transfusion of packed red blood cells was given during surgery, and the secondary outcome was the volume and type of fluids administered during surgery.

\section{Statistical analysis}

The data were analyzed with statistical software (IBM SPss Statistics for Windows, Version 21.0, IBM Corp., Armonk, NY). Univariate comparisons were made with $t$-test and Pearson's $\chi^{2}$ test (chi-square test), where appropriate for variable scale. Variables found to be significantly associated univariately with need for transfusion of packed red blood cells during surgery were entered into a binary logistic regression procedure using a backward conditional method. 67 patients were included in the logistic regression model, 50 who received packed red blood cells in comparison with 17 who did not. A series of regression procedures was run, and the final model is table reported which includes variables significantly predictive at a $P$-value of $\leq 0.05$.

\section{Results}

In the 206 patients who had the diagnosis of necrotizing entercolitis, 88 patients $(43 \%)$ had exploratory laparotomy with bowel resection (67 patients) or without bowel resection (21 patients). Packed red blood cells were given during surgery more frequently to patients who had bowel resection than patients who did not have bowel resection (Table 1). Patients who received packed red blood cells during surgery had longer mean duration of surgery, lower preoperative hemoglobin and hematocrit, higher postoperative hemoglobin and hematocrit, more platelet transfusions, and greater estimated blood loss than patients who did not receive packed red blood cells during surgery (Table 1). The use of crystalloid, colloid, and fresh frozen plasma was similar between patients

Table 1 Clinical characteristics of patients who had laprotomy for NEC with and with out PRBCs given ${ }^{a}$

\begin{tabular}{|c|c|c|c|}
\hline Variable & No & Yes & $P \leq^{b}$ \\
\hline Gestational age at birth (weeks) & $29 \pm 4$ & $30 \pm 4$ & 0.318 \\
\hline Body weight at birth (kg) & $1.2 \pm 0.8$ & $1.3 \pm 0.7$ & 0.464 \\
\hline Age at surgery (d) & $35 \pm 57$ & $27 \pm 25$ & 0.452 \\
\hline Body weight at surgery $(\mathrm{kg})$ & $2 \pm 1$ & $1.8 \pm 0.9$ & 0.697 \\
\hline Duration of surgery (min) & $106 \pm 44$ & $153 \pm 41$ & 0.01 \\
\hline \multicolumn{4}{|l|}{ Bowel resection performed } \\
\hline Yes & $17(25)$ & $50(75)$ & 0.01 \\
\hline No & $20(95)$ & $1(5)$ & \\
\hline \multicolumn{4}{|l|}{ Hemoglobin $\left(\mathrm{g} \cdot \mathrm{dl}^{-1}\right)$} \\
\hline Preoperative & $13 \pm 2$ & $12 \pm 2$ & 0.01 \\
\hline Postoperative & $12 \pm 3$ & $14 \pm 3$ & 0.01 \\
\hline \multicolumn{4}{|l|}{ Hematocrit (\%) } \\
\hline Preoperative & $38 \pm 7$ & $34 \pm 5$ & 0.01 \\
\hline Postoperative & $34 \pm 7$ & $41 \pm 8$ & 0.01 \\
\hline \multicolumn{4}{|l|}{ Platelet count $\left(\times\left. 10^{9} \cdot\right|^{-1}\right)$} \\
\hline Preoperative & $164 \pm 104$ & $184 \pm 136$ & 0.45 \\
\hline Postoperative & $166 \pm 110$ & $122 \pm 87$ & 0.071 \\
\hline \multicolumn{4}{|l|}{ Transfusions during surgery } \\
\hline Packed red blood cells $\left(\mathrm{ml} \cdot \mathrm{kg}^{-1}\right)$ & $0 \pm 0$ & $32 \pm 30$ & - \\
\hline Crystalloid $\left(\mathrm{ml}^{\prime} \mathrm{kg}^{-1}\right)$ & $28 \pm 24$ & $37 \pm 39$ & 0.216 \\
\hline Colloid $\left(\mathrm{ml} \cdot \mathrm{kg}^{-1}\right)$ & $15 \pm 14$ & $21 \pm 16$ & 0.069 \\
\hline Fresh frozen plasma $\left(\mathrm{ml} \cdot \mathrm{kg}^{-1}\right)$ & $1 \pm 6$ & $5 \pm 17$ & 0.132 \\
\hline Platelets $\left(\mathrm{ml} \cdot \mathrm{kg}^{-1}\right)$ & $0.6 \pm 2$ & $8 \pm 15$ & 0.01 \\
\hline Estimated blood loss $\left(\mathrm{ml} \cdot \mathrm{kg}^{-1}\right)$ & $3 \pm 3$ & $15 \pm 19$ & 0.01 \\
\hline
\end{tabular}

${ }^{a} N=88$ patients (bowel resection, 67 patients; no bowel resection, 21 patients). Data reported as mean \pm SD or number (\%).

${ }^{b} N S$, not significant $(P>0.05)$. 
who received or did not receive packed red blood cells during surgery (Table 1). There was no difference in gestational age or body weight at birth, age or body weight at surgery, or preoperative and postoperative platelet count between patients who received or did not receive packed red blood cells during surgery (Table 1).

We were able to find additional information on 15 of the patients that were operated without receiving a bowel resection. Ten of the patients had pan-intestinal necrosis, and no further surgery was indicated. The other five patients had operations, which did not include a bowel resection: Three patients had proximal diversion for ischemic, nonnecrotic bowel; one patient had evacuation of inspissated meconium; and one patient a Ladd procedure for malrotation.

Variables significantly associated univariately with the need for packed red blood cells were further explored using the logistic regression procedure. These included duration of surgery in minutes, hemoglobin both preoperative and postoperative, hematocrit both preoperative and postoperative, platelet count both preoperative and postoperative, need for platelet transfusion during surgery, and estimated blood loss. After a series of runs which excluded nonsignificant variables, the final model included two significantly predictive variables: preoperative hemoglobin and postoperative hemoglobin (Table 2). On the average, a one unit change in preoperative hemoglobin resulted in an odds of 0.552 (95\% CI: 0.364-0.836) for need of packed red blood cells, while a one unit change in postoperative hemoglobin revealed a 2.0 -fold increase (95\% CI: 1.335-2.993). Model data fit was examined using the Hosmer-Lemeshow test, which assesses whether or not the observed event rates match expected event rates in the subgroups of the model population. The model showed no evidence of lack of fit based upon an obtained $\mathrm{H}-\mathrm{L}$ significance value of 0.251 .

\section{Discussion}

Optimal anesthetic management of neonates in emergency surgery is important to anesthesiologists. Surgery for NEC in small babies imposes critical important decision-making scenarios.

Univariately, our study found that intraoperative transfusion of packed red blood cells should be considered when presented with a low preoperative hemoglobin, a need for bowel resection, and low preoperative hematocrit. The association of bowel resection and transfusion requirement may be a result of the increased blood flow to the bowel affected by necrotizing enterocolitis and the systemic inflammatory condition associated with fluid retention. Preoperative anemia in these patients may be attributed to sepsis and multiple diagnostic blood draws. Increased transfusion with duration of surgery is likely due to a more complicated clinical case.

While this information seems obvious, our data mirror current clinical practice. Ongoing resuscitation in the NICU prior to bringing the patient in the operating room may play a role in this decision-making process. Blood products should be available in the operating room for these operations because urgent blood transfusion may be required. Furthermore, these operations frequently occur during the evening or night, when logistical issues may delay the availability of blood products. As most hospitals require that blood products be prepared for NEC neonates about to undergo surgery, this model serves to validate the legitimacy of the current clinical procedure.

Previous studies have suggested that packed red blood cells should be available for operations with estimated blood loss $>10 \%$ of total blood volume and hematocrit should be maintained $>30 \%(12,13)$.

The secondary outcomes of our findings include albumin use in patients with NEC and blood transfusion. Albumin is frequently the primary colloid-based plasma expander selected for use in neonates and infants and was the first choice for fluid resuscitation in the study population (8-11). Our data report the use of albumin and other resuscitation fluids in our patient population.

Intraoperative decisions for fluid resuscitation frequently are based on close observation of the surgical procedure and vital signs. The patients who did not require a transfusion often had higher hemoglobin concentrations at the beginning of surgery than those that did require a transfusion, and the postoperative hematocrit levels was still $>30 \%$.

\section{Limitations}

Limitations of the present study include a retrospective study design, and the exact circumstances about the decisions to transfuse were not always available from the medical or anesthesia records. Decisions to transfuse are likely to have been based on empiric clinical judgment in response to major hemodynamic changes, absence of point-of-care measurements of hematocrit, and limited vascular access for blood sampling. In addition, only the cumulative amount of each blood product was recorded, which limited the availability of specific details for analysis. Some of the patients did receive blood products other than PRBCs during bowel resection. There was no description on the anesthesia chart about the need for such products but likely these 
Table 2 Logistic regression final model examining risk factors for the need for PRBC

\begin{tabular}{|c|c|c|c|c|c|c|c|c|}
\hline & B & SE & Wald & $d f$ & Sig. & $\operatorname{Exp}(B)$ & $\begin{array}{l}95 \% \\
\mathrm{Cl} \text { lower }\end{array}$ & $\begin{array}{l}95 \% \\
\mathrm{Cl} \text { upper }\end{array}$ \\
\hline Pre-Op Hb & -0.595 & .212 & 7.881 & 1 & 0.05 & 0.552 & 0.364 & 0.836 \\
\hline Post-Op Hb & 0.693 & .206 & 11.304 & 1 & 0.001 & 1.999 & 1.335 & 2.993 \\
\hline Constant & -0.537 & 2.671 & 0.40 & 1 & 0.841 & 0.584 & & \\
\hline
\end{tabular}

patients received these products as a continuation of NICU management.

\section{Conclusion}

In conclusion, lower preoperative hemoglobin with need for bowel resection indicates a possible requirement for blood transfusion. The logistic regression model (Table 2) may provide a useful preoperative prediction of the need for packed red blood cells during surgery. Given the exploratory, and unbalanced, sample sizes used in this study, the results provided should be interpreted with caution. Therefore, additional appropriately powered prospective trials are needed to validate this result. The contribution of this study may further assist clinicians in making a decision to perform a blood transfusion in these patients.

\section{Acknowledgments}

None of the authors report any financial conflicts. Local Human Investigation Committee approved study. Sincere thanks to Maria Zestos M.D. and Benjamin Guslits M.D., F.R.C.P.C., M.B.A. for their support and help.

\section{Funding}

This research was carried without funding.

\section{Conflict of interest}

No conflicts of interest declared.

\section{References}

1 Neu J, Walker WA. Necrotizing enterocolitis. N Engl J Med 2011; 364: 255-264. doi:10s056/NEJMra1005408.

2 Henry MCW, Moss RL. Necrotoizing enterocolitis. In: Holcomb GW III, Murphy JP, Ostlie DJ, eds. Ashcraft's Pediatric Surgery, 5th edn. Philadelphia, PA: Saunders Elsevier, 2010: 439-455.

3 Henry MC, Moss RL. Neonatal necrotizing enterocolitis. Semin Pediatr Surg 2008; 17: 98-109.

4 Moss RL, Dimmitt RA, Henry MC et al. A meta-analysis of peritoneal drainage versus laparotomy for perforated necrotizing enterocolitis. J Pediatr Surg 2001; 36: 1210-1213.

5 Rao SC, Basani L, Simmer K et al. Peritoneal drainage versus laparotomy as initial surgical treatment for perforated necrotizing enterocolitis or spontaneous intestinal perforation in preterm low birth weight infants. Cochrane Database Syst Rev 2011; 6 CD006182.

6 Guthrie SO, Gordon PV, Thomas V et al. Necrotizing enterocolitis among neonates in the United States. J Perinatol 2003; 23: 278-285.

7 Lin PW, Stoll BJ. Necrotising enterocolitis. Lancet 2006; 368: 1271-1283.

8 Murat I, Dubois MC. Perioperative fluid therapy in pediatrics. Pediatr Anesth 2008; 18: $363-370$.

9 Bailey AG, McNaull PP, Jooste E et al. Perioperative crystalloid and colloid fluid management in children: where are we and how did we get here? Anesth Analg 2010; 110: 375-390.

10 Schwarz U. Intraoperative fluid therapy in infants and young children [in German]. Anaesthesist 1999; 48: 41-50.

11 Söderlind M, Salvignol G, Izard P et al. Use of albumin, blood transfusion and intraoperative glucose by APA and ADARPEF members: a postal survey. Paediatr Anaesth 2001; 11: 685-689.

12 Peiris K, Fell D. The prematurely born infant and anaesthesia. Continuing Education in Anaesthesia. Critical Care Pain 2009; 9: 73-77.

13 Canadian Paediatric Society. Red blood cell transfusions in newborn infants: revised guidelines. Paediatr Child Health 2002; 7 : 553-566. 